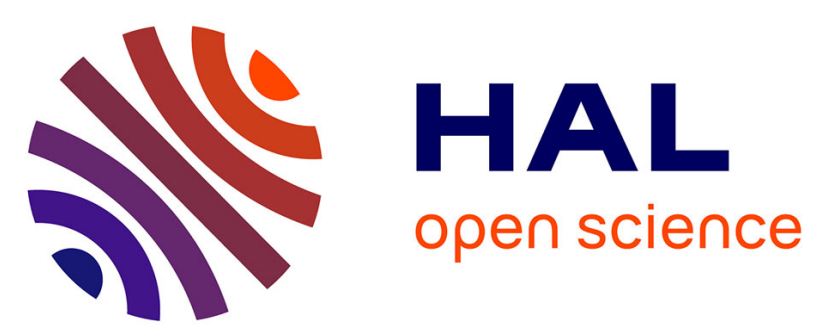

\title{
Individual vs. Global Radio Resource Management in a Hybrid Broadband Network
}

Kinda Khawam, Marc Ibrahim, Johanne Cohen, Samer Lahoud, Samir Tohmé

\section{To cite this version:}

Kinda Khawam, Marc Ibrahim, Johanne Cohen, Samer Lahoud, Samir Tohmé. Individual vs. Global Radio Resource Management in a Hybrid Broadband Network. [Research Report] PI-1956, 2010, pp.9. inria-00528575

\section{HAL Id: inria-00528575 \\ https://hal.inria.fr/inria-00528575}

Submitted on 22 Oct 2010

HAL is a multi-disciplinary open access archive for the deposit and dissemination of scientific research documents, whether they are published or not. The documents may come from teaching and research institutions in France or abroad, or from public or private research centers.
L'archive ouverte pluridisciplinaire HAL, est destinée au dépôt et à la diffusion de documents scientifiques de niveau recherche, publiés ou non, émanant des établissements d'enseignement et de recherche français ou étrangers, des laboratoires publics ou privés. 


\title{
Individual vs. Global Radio Resource Management in a Hybrid Broadband Network
}

\author{
Kinda Khawam ${ }^{*}$, Marc Ibrahim ${ }^{* *}$ Johanne Cohen $^{* * *}$, Samer Lahoud ${ }^{* * * *}$, Samir Tohme ${ }^{* * * * *}$ \\ Contact author: kinda.khawam@prism.uvsq.fr
}

\begin{abstract}
Nowadays, with the abundance of diverse air interfaces in the same operating area, advanced Radio Resource Management (RRM) is vital to take advantage of the available system resources. In such a scenario, a mobile user will be able to connect concurrently to different wireless access networks. In this paper, we consider the downlink of a hybrid network with two broadband Radio Access Technologies (RAT): WiMAX [1] and WiFi [2]. Two approaches are proposed to load balance the traffic of every user between the two available RATs: an individual approach where mobile users selfishly strive to improve their performance and a global approach where resource allocation is made in a way to satisfy all mobile users. We devise for the individual approach a fully distributed resource management scheme portrayed as a non-cooperative game. We characterize the Nash equilibriums of the proposed RRM game and put forward a decentralized algorithm based on replicator dynamics to achieve those equilibriums. In the global approach, resources are assigned by the system in order to enhance global performances. For the two approaches, we show that after convergence, each user is connected to a single RAT which avoids costly traffic splitting between available RATs.
\end{abstract}

Key-words: Non-cooperative game theory, non-linear optimisation, WiMAX, WiFi, 4G networks.

\section{Approches Individuelle et globale pour la gestion des ressources radio dans un réseau large-bande hybride}

Résumé : $\quad$ Actuellement, avec la diversification des interfaces radio présentes dans une même zone géographique, il devient vital de mettre en place des techniques avancées de gestion de ressources radio afin de profiter des ressources disponibles. Dans un tel scénario, un utilisateur mobile peut se connecter simultanément à différents réseaux d'accès sans-fil. Dans ce papier, nous considérons la voie descendante d'un réseau hybride avec deux technologies d'accès radio large-bande disponibles: WiMAX [1] et WiFi [2]. Deux approches sont proposées pour équilibrer le trafic de chaque utilisateur sur les deux technologies d'accès: une approche individuelle où les mobiles cherchent à améliorer leurs performances d'une manière égoste et une approche globale où l'allocation de ressources est faite de façon à satisfaire tous les utilisateurs mobiles. Nous introduisons pour l'approche individuelle un schéma de gestion distribuée des ressources formulée en un jeu non-coopératif. Nous caractérisons les équilibres de Nash du jeu proposé et proposons un algorithme distribué pour atteindre ces équilibres. Pour l'approche globale, les ressources sont allouées par le système dans le but d'améliorer les performances globales. Pour les deux approches, nous démontrons qu'après convergence, chaque utilisateur se trouve connecté à une seule technologie d'accès sans-fil, ce qui permet d'éviter un partage de trafic coûteux entre les technologies disponibles.

Mots clés : Théorie des jeux non-coopératifs, optimisation non-linéaire, WiMAX, WiFi, réseaux 4G.

\footnotetext{
* Université de Versailles - PRISM Laboratory, France

** Ecole Supérieure des Ingenieurs de Beyrouth, Lebanon

*** Université de Versailles - PRISM Laboratory, France

**** University of Rennes I - IRISA, France

****** Université de Versailles - PRISM Laboratory, France
} 


\section{Introduction}

The migration of wireless networking towards the $4 \mathrm{G}$ era is distinguished by the proliferation of radio access technologies. As no existing technology can be surrogated by another one, the coexistence of today wireless networks is the best solution at hand to deal with the incessantly growing user demands. Hence, in this composite radio environment, mobile users will be able to simultaneously utilize services through diverse RATs. The RAT selection policy is crucial and must be designed astutely to avoid resource wastage. Furthermore, it can be either maneuvered in an individual fashion giving precedence to the user interests or in a global fashion where the interests of the entire mobile users are prevalent. In the individual approach, each user aims to minimize selfishly its own cost. In this competitive environment, resorting to non-cooperative game theory is natural in order to obtain optimal traffic allocation among the different RATs. Hence, users are intelligent terminals that can discover the radio environment and connect to available RATs (i.e., radio cognitive). In this sense, we present a distributed game-theoretic RRM framework for wireless access for the downlink channel of a double RAT network. In the global approach, we resort to non-linear optimization to minimize global network cost. We prove that, for both approaches, optimal load balancing between RATs boils down to a unique RAT selection, thus avoiding therefore expensive frequent swaps between RATs. In fact, the different technologies may have different delays, packet sizes or coding systems. Hence, re-constructing messages sent via two RATs may be hazardous.

Results are validated through extensive simulations of several implementations of both approaches in the practical setting of a geographical area covered by a global WiMAX network overlapping with several local WiFi hotspots.

RRM in hybrid networks is an emerging area of research and few related work is available in the literature. In $([3,4,5,6])$, noncooperative game theory is used for RRM. In [3], the available bandwidth is shared in a distributed manner between hybrid networks. The centralized approach is based on a two-player finite game that is played by access networks. In [4], users in a cell are divided into two groups depending on their location in the cell. The RRM scheme is conducted as a finite two-player game played among these two groups which induces some kind of collaboration between mobile users. In [5], the selection between WiFi and UMTS is formulated as an interesting symmetric non-cooperative game that reduces to a threshold policy. Our work was done independently of the work in [6] which is very different, despite the apparent similarities (load balancing between WiMAX and WiFi and the use of replicator dynamics). It relies on an approach mixing the individual and global tendencies. In [7], another mixed approach assigns incoming users to a hybrid WiMAX-UMTS network based on the Semi Markov Decision Process theory.

The rest of the paper is organized as follows. The system model is described in Section 2. The individual approach apprehended as a non-cooperative game is described in Section 3, where a distributed learning algorithm is proposed. The global approach is sketched in 4. Numerical results are presented in 5. Conclusions are given in Section 6.

\section{Hybrid System Model on the Downlink}

A WiMAX cell ray is in the order of a few kilometers while the WiFi cell ray spans from a few tens to a few hundreds of meters only. Hence, typically, a WiMAX cell will be covered with several WiFi hotspots. We consider a geographic region that is entirely covered by a WiMAX base station (BS) and partly covered by WLAN Access Points (AP) as in Fig. 1. Only mobile users present in WiFi hotspots benefit from load balancing their traffic between the two technologies. The other mobile users profit only from the WiMAX coverage. In the remaining we use the generic term cell to designate the WiMAX cell or a WiFi hotspot.

In what follows, we will present our model in terms of traffic and data rate and finally define a suitable cost function.

\subsection{Network Structure}

The index $x=0 . . H$ is used throughout the paper to designate a given cell, $x=0$ indexing the WiMAX cell and $x=1 . . H$ referring to the $H$ WiFi hotspots. We denote by $n_{x}$ the number of users able to connect to cell $x$. Since all users can connect to the WiMAX cell, $n_{0}$ is the total number of mobile users and $\bar{n}=n_{0}-\sum_{x=1}^{H} n_{x}$ is the number of users that are only serviced by WiMAX. Thus, only $n=\sum_{x=1}^{H} n_{x}$ users are engaged in the RAT selection; such users will be indexed by $k=1 . . n$ and will be coined hybrid users. We term by $h(k)$ the hotspot of user $k$.

\subsection{Traffic Model}

The goal of the article is to appropriately allocate the traffic of hybrid mobile users between the available RATs. For that, we denote by $\theta_{k}$ the instantaneous fraction of time during which user $k$ is assigned to its hotspot $h(k)$ (or the percentage of its traffic placed on the hotspot). Hence, $1-\theta_{k}$ is the remaining fraction of time spent by user $k$ in the WiMAX cell. 


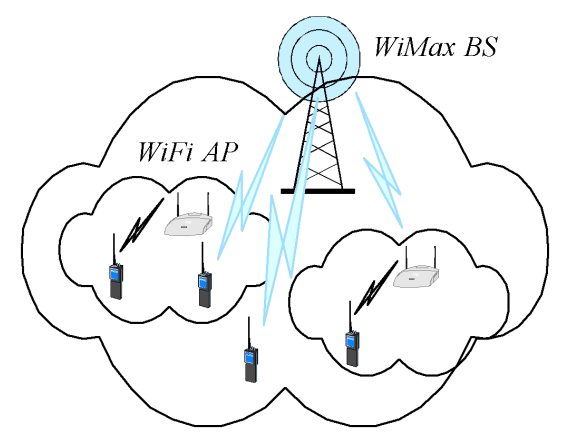

Figure 1: Hybrid wireless access environment

\subsection{System Model}

The radio conditions of a mobile user $k$ infer the instantaneous peak rate it can obtain when connected to a given cell. Those conditions are assumed to be invariant as neither mobility nor fading are taken into account. Let $\chi_{k, x}$ denote the instantaneous peak rate of user $k$ in cell $x$. Each hybrid user perceives two peak rates $\chi_{k, 0}$ and $\chi_{k, h(k)}$ depending on the chosen RAT.

\subsubsection{Data Rate in the WiMAX Cell}

The wireless resource is time-shared between all the $n_{0}$ mobile users. We consider a Fair Time sharing model where active users are given the same chance to access resources [1]. Hence, the data rate of user $k$ assigned to WiMAX is:

$$
\begin{aligned}
R_{k, 0} & =\frac{\chi_{k, 0}(t)}{\sum_{i=1}^{n_{0}} \mathbb{1}_{\{\text {user i admitted in WiMAX }\}}} \\
& =\frac{\chi_{k, 0}}{1+\bar{n}+\sum_{i \neq k}^{n} \mathbb{1}_{\{\text {user i admitted in WiMAX }\}}}
\end{aligned}
$$

\subsubsection{Data Rate in the WiFi Hotspots}

In this paper, the uplink traffic is neglected which leads to a fair access scheme on the downlink channel. However, when a low rate user captures the channel, it will use it for a long time, which penalizes high rate users and reduces the fair access strategy to a case of fair rate sharing ${ }^{1}$. Thus, the data rate of user $k$ in hotspot $h(k)$ is:

$$
R_{k, h(k)}=1 / \sum_{\substack{i \\ h(i)=h(k)}} \frac{\mathbb{1}_{\{\text {user i admitted in hotspot } \mathrm{h}(\mathrm{k})\}}}{\chi_{i, h(i)}}
$$

\subsection{Cost Function}

The objective of the present traffic allocation is to set the amount of traffic that every user should convey through each RAT so that all mobile users are satisfied. Satisfaction for a mobile user is defined here as the minimization of the cost it perceives subsequent to a given RAT selection. The cost function adopted is an image of service time.

We denote by $T_{k, x}$ the amount of time necessary to send a data unit in cell $x$. In WiMax, we deduce from (1) the following:

$$
T_{k, 0}=\mathbb{E}\left[1 / R_{k, 0}\right]=\frac{1+\bar{n}+\sum_{i=1, i \neq k}^{n}\left(1-\theta_{i}\right)}{\chi_{k, 0}}
$$

\footnotetext{
${ }^{1}$ Assuming no collisions and a constant MAC frame size and neglecting the 802.11 waiting times (i.e., DIFS, SIFS, ...) in comparison with transmission times.
} 
The same applies in hotspot $h(k)$ using formula (2):

$$
T_{k, h(k)}=\mathbb{E}\left[1 / R_{k, h(k)}\right]=\frac{1}{\chi_{k, h(k)}}+\sum_{\substack{i=1, i \neq k \\ h(i)=h(k)}} \frac{\theta_{i}}{\chi_{i, h(i)}}
$$

Therefore, the cost function of a hybrid user $k$, defined as its expected time necessary to send a unit of data in this hybrid environment, is given by the following:

$$
C_{k}=T_{k, 0} \cdot\left(1-\theta_{k}\right)+T_{k, h(k)} \cdot \theta_{k}
$$

In the individual approach, we formulate a non-cooperative game among hybrid users where the objective of each user $k$ is to minimize its individual cost function $C_{k}$; while in the global approach, the system load balances the traffic of each hybrid user among RATs in order to minimize the total network cost, defined as the sum of all individual costs.

\section{Individual Approach}

Game theory models the interactions between players competing for a common resource. Therefore, this tool is adapted to radio resource management modeling. For each state of the system, defined by the number of hybrid users $n$, we define a multi-player non-cooperative game $G$ between those $n$ mobile users present in a double coverage area.

In this model, there is a sequence of one-stage games, each corresponding to a given state of the system, defined by the number of hybrid users. Whenever a new hybrid mobile is admitted in the system, the game is played again with an additional player. We assume that mobiles have complete information on each other. Mobile users are assumed to make their decisions without knowing the decisions of each other.

The formulation of this non-cooperative game $G=\langle N, S, C\rangle$ can be described as follows:

- A finite set of players: $N=(1, \ldots, n)$.

- The space of pure strategies $S$ formed by the Cartesian product of each set of pure strategies $S=S_{1} \times S_{2} \times \ldots \times S_{n}$. An action of a user $k$ is the proportion of traffic $\theta_{k}$ sent via the hotspot $h(k)$. Hence $S_{k}=[0,1]$. The strategy chosen by player $k$ is then $\theta_{k}$ while $\theta_{-k}$ denotes the set of actions taken by all other players. A strategy profile $\theta=\left(\theta_{1}, \ldots, \theta_{n}\right)$ specifies the strategies of all players

- A set of cost functions $C=\left(C_{1}(\theta), C_{2}(\theta), \ldots, C_{n}(\theta)\right)$ that quantify players' costs for a given strategy profile $\theta$.

\subsection{Nash Equilibrium}

In a non-cooperative game, an efficient solution is that where all players adhere to a Nash Equilibrium (NE) which is a profile of strategies in which no player will take advantage by deviating its strategy unilaterally. Hence, it is a strategy profile $\theta^{*}$ where each player's strategy is an optimal response to the other players' strategies:

$$
C_{k}\left(\theta^{*}\right)=C_{k}\left(\theta_{k}^{*}, \theta_{-k}^{*}\right) \leq C_{k}\left(\theta_{k}{ }^{\prime}, \theta_{-k}^{\prime}\right), \forall k \in N, \forall \theta_{k}^{\prime} \in S_{k}
$$

For every $k, C_{k}$ is convex w.r.t. $\theta_{k}$ and continuous w.r.t. $\theta_{i}, i \neq k$. Hence, a Nash equilibrium exists [8].

For sake of simplicity, we re-write the cost function as follows:

$$
C_{k}(\theta)=\theta_{k} \cdot f_{k}\left(\theta_{-k}\right)+g_{k}\left(\theta_{-k}\right)
$$

where

$$
f_{k}\left(\theta_{-k}\right)=\frac{1}{\chi_{k, h(k)}}+\sum_{\substack{i=1, i \neq k \\ h(i)=h(k)}} \frac{\theta_{i}}{\chi_{i, h(i)}}-\frac{n_{0}-\sum_{i=1, i \neq k}^{n} \theta_{i}}{\chi_{k, 0}}
$$

and

$$
g_{k}\left(\theta_{-k}\right)=\frac{n_{0}-\sum_{i=1, i \neq k}^{n} \theta_{i}}{\chi_{k, 0}}
$$

Proposition 1. The Nash equilibrium is either the solution of the following system of $n$ equations :

$$
f_{k}\left(\theta_{-k}\right)=0, \quad \forall k \in N
$$

or at the boundaries of the strategy space. 
Proof. Since the cost functions are convex, at the Nash equilibrium, the $\theta_{k}$ are obtained by computing the partial derivative of the cost function of each player in respect to its action $\theta_{k}$ and by equating the result to zero.

$$
\frac{\partial C_{k}}{\partial \theta_{k}}=f_{k}\left(\theta_{-k}\right)=0, \forall k \in N
$$

We obtain a system of $n$ equations with $n$ unknowns given in (8).

Unfortunately, the solution of the above system is not always feasible (not between 0 and 1 ) as the set of actions is bounded. In such a case, according to 6 and $\forall \theta_{k}{ }^{\prime} \in S_{k}$, we have what follows:

$$
\begin{aligned}
& \theta_{k} \cdot f_{k}\left(\theta_{-k}\right)+g_{k}\left(\theta_{-k}\right) \leq \theta_{k}{ }^{\prime} \cdot f_{k}\left(\theta_{-k}\right)+g_{k}\left(\theta_{-k}\right) \Rightarrow \\
& \theta_{k} \cdot f_{k}\left(\theta_{-k}\right) \leq \theta_{k}{ }^{\prime} \cdot f_{k}\left(\theta_{-k}\right) \Rightarrow \\
& \quad \text { If } f_{k}\left(\theta_{-k}\right)>0 \Rightarrow \theta_{k} \leq \theta_{k}{ }^{\prime}, \forall \theta_{k}{ }^{\prime} \in S_{k} \Rightarrow \theta_{k}=0 \\
& \quad \text { If } f_{k}\left(\theta_{-k}\right)<0 \Rightarrow \theta_{k} \leq \theta_{k}, \forall \theta_{k}{ }^{\prime} \in S_{k} \Rightarrow \theta_{k}=1 \\
& \text { If } f_{k}\left(\theta_{-k}\right)=0 \Rightarrow \theta_{k} \text { can take any value in }[0,1]
\end{aligned}
$$

We notice that if $f_{k}\left(\theta_{-k}\right)$ is negative for a user $k \in N$, this user always favors WiFi to WiMAX by setting $\theta_{k}=1$. This is because $f_{k}<0$ implies $T_{k, h(k)}<T_{k, 0}$, hence the cost in WiFi is always lower than that endured in WiMAX. The same idea is valid for the inverse situation $\left(f_{k}\left(\theta_{-k}\right)>0\right)$.

\subsubsection{Exhaustive Search Algorithm to compute a boundary NE}

We can see from 9, that if for user $k, f_{k}\left(\theta_{-k}\right) \neq 0$ (then $\theta_{k}=\{0,1\}$ ) while for the remaining users $j \neq k$, we have $f_{j}\left(\theta_{-j}\right)=0$ : the system of $n$ equations with $n$ unknowns reduces to $n-1$ equations with $n-1$ unknowns. In fact, we get two systems of equations: one where $\theta_{k}=0$ and one where $\theta_{k}=1$. If any of these two systems gives a feasible solution, then it is a NE. This is done recursively for $i=1 \ldots n$ (giving $2 \times n$ sets of equations to solve). We then consider the case where $f_{k} \neq 0$ for only two users. Then 9 reduces to $n-2$ equations with $n-2$ unknowns (in fact, we have to solve $C_{n}^{2} \times 2^{2}$ sets of equations). Any set with a feasible solution gives a new NE. This reasoning is iterated in order to consider all possibilities.

\subsubsection{Iterative Search Algorithm to compute NE}

As explained in the previous paragraph 3.1.1, obtaining the various Nash equilibriums is computationally intensive. Thus, we apply an iterative search algorithm that is easy to implement.

With a boundary starting point, e.g. $\theta_{k}(0)=1$ for $k=1, . ., n$, the algorithm iterates until convergence, that is $\theta_{k}(t)=\theta_{k}(t-1)$ ) for all $k \in N$.

Input: $\theta_{k}(0)$ for $k=1, . ., n$ and $t=1 . \theta_{k}(0)$ can be 0 or 1 .

1. At time $\mathrm{t}$, for $k=1, . ., n$

Do compute $f_{k}\left(\theta_{1}(t), . ., \theta_{k-1}(t), \theta_{k+1}(t-1), . ., \theta_{n}(t-1)\right) \Rightarrow \theta_{-k}(t)$ is set according to 9 .

In particular, $\theta_{k}(t)=\theta_{k}(t-1)$ if $f_{k}\left(\theta_{-k}\right)=0$.

2. Do $k=k+1$

3. if $\left(\theta_{k}(t)=\theta_{k}(t-1)\right) \leq \epsilon$ for all $k$, Output: $\theta_{k}(t)$ for $k=1, . ., n$. else Do $t=t+1$ and Go to step 1 .

Extensive simulations carried out show the convergence of the proposed algorithm in 3.1.2 as results match those obtained in 3.1.1.

\subsection{Distributed Learning of NE}

In a real environment, an iterative algorithm as the one proposed in 3.1.2 cannot be practically applied as every user needs to know the policy of all other users present in the cell which necessitates expensive signaling and hinders the benefits of a distributed resource management policy. As a consequence, we fall back to the proposed algorithm in [11] to learn Nash equilibriums. In the cited paper, players have pure strategies and the devised algorithm will give the mixed strategies corresponding to a probability distribution over pure strategies.

In the present context, this algorithm can be applied by considering the discrete version of our problem: hybrid users have a finite set of strategies, each strategy amounts to selecting only one RAT (the special case where $\theta_{k}=\{0,1\}$ ). Therefore, the set of pure strategies of any hybrid user $k$ in the finite game, termed $s_{k}$, is $s_{k}=\{h(k), 0\}$ corresponding to choosing WiMAX or WiFi hotspot $h(k)$ respectively. The hybrid user gets thereby the following cost: 


$$
c_{k}=\left\{\begin{array}{ccc}
T_{k, h(k)} & \text { if } & s_{k}=0 \\
T_{k, 0} & \text { if } & s_{k}=h(k)
\end{array}\right.
$$

Thus, a mixed strategy matches our probability distribution $\left(\theta_{k}, 1-\theta_{k}\right)$ on $s_{k}$.

The general distributed algorithm is the following:

Input: $\theta(0)=\left(\theta_{1}(0), \ldots, \theta_{n}(0)\right)$ any vector of probabilities.

At each round $t$, every hybrid user $k$ :

- selects the WiFi hotspot with probability $\theta_{k}(t)$. This leads to an outcome $r_{k}$ for hybrid user $k$.

- Updates $\theta_{k}(t)$ as follows:

$$
\theta_{k}(t+1)=\theta_{k}(t)+b \cdot F_{k}^{b}\left(r_{k}(t), s_{k}(t), \theta_{k}(t)\right)
$$

In the general version of the depicted algorithm, the function $F_{k}^{b}\left(r_{k}(t), s_{k}(t), \theta_{k}(t)\right)$ can be very broad (some conditions are nevertheless defined in [11]) and the cost $r_{k}(t)$ (obtained at step $t$ by user $k$ as a consequence of selected strategy) can be completely random.

Algorithms of this form are fully distributed as decisions made by users are completely decentralized: at each time step, hybrid user $k$ only needs to know $r_{k}$ and $\theta_{k}$, that is to say its current cost and mixed strategy, to update its own mixed strategy. In our model, $r_{k}$ is not random but equal to $1-c_{k}$.

\subsubsection{Replicator Dynamics}

We are interested in a particular family of these algorithms termed Replicator Dynamics because they can converge in our proposed scheme to Nash equilibriums. For that case, function $F_{k}^{b}$ is given by:

$$
\begin{aligned}
& F_{k}^{b}\left(r_{k}(t), s_{k}(t), \theta_{k}(t)\right)= \\
& \gamma\left(r_{k}(t)\right) \cdot\left(1-\mathbb{1}_{\left\{s_{k}=h(k)\right\}} \cdot \theta_{k}(t)\right)
\end{aligned}
$$

where $\gamma: R \rightarrow[0,1]$ is some affine increasing function. The proof of convergence of this replicator is given in Appendix as well as the proof of the following proposition:

Proposition 2. For any initial condition where $\forall i \in N, \theta_{i}^{x} \neq 0$ or $\theta_{i}^{x} \neq 1$, the considered learning algorithm converges to a pure Nash equilibrium.

Thus, according to proposition 2, optimal load balancing between RATs reverts to a sole RAT selection, whereas each hybrid mobile user is assigned to a single RAT. Consequently, costly recurrent shifts between RATs are eschewed.

\section{Global Approach}

Contrary to the aforementioned individual approach where precedence is given to the individual user interests, resource allocation may be performed in a way to privilege the overall system performance. This illustrates the case where a system operator chooses to optimize radio resources considering the interest of all mobile users. We capture this case by introducing a global approach, where the system assigns the traffic of each hybrid mobile user among RATs in order to minimize the total network cost. The network cost, denoted by $C_{t}$, is defined as the sum of mobile users individual costs and given by: $C_{t}\left(\theta_{k}, k \in N\right)=\sum_{k=1}^{n} C_{k}\left(\theta_{k}\right)$, where $N$ denotes the set of hybrid users. Thus, the global approach can be formulated as an optimization problem $(\mathcal{P})$ introduced hereafter:

$$
(\mathcal{P}) \text { : Minimize } C_{t}\left(\theta_{k}, k \in N\right)=\sum_{k=1}^{n} C_{k}\left(\theta_{k}\right)
$$

Subject to: $0 \leq \theta_{k} \leq 1, k=1, . ., n$

\subsection{Optimal Solution for the Global Approach}

Problem $(\mathcal{P})$ is a non-linear optimization that consists in minimizing the objective function $C_{t}\left(\theta_{k}, k \in N\right)$ over the convex polytope $\left\{0 \leq \theta_{k} \leq 1, k=1, \ldots, n\right\}$. The vertices of this polytope correspond to the points where $\theta_{k}=0$ or $\theta_{k}=1$ for each $k=1, . ., n$. Theorem 3 demonstrates that the global optimal solution for Problem $(\mathcal{P})$ is always reached on the vertices of the polytope.

Theorem 3. A global optimal solution to Problem $(\mathcal{P})$ is reached on the vertices of the convex polytope. 
Proof. Suppose that a global optimal solution to $\operatorname{Problem}(\mathcal{P})$ is reached for $\hat{\theta}=\left(\hat{\theta}_{k}, k=1, \ldots, n\right)$. Let us proceed by contradiction and suppose that there exists an integer $j$ with $1 \leq j \leq n$ for which $\hat{\theta}_{j} \notin\{0,1\}$. The function $C_{t}\left(\theta_{j}\right):=C_{t}\left(\theta_{j}, \theta_{k}=\hat{\theta}_{k}, k \in N-\{j\}\right)$ is obtained by taking $\theta_{k}=\hat{\theta}_{k}, i \in N-\{j\}$ as a linear function of variable $\theta_{j}$. Trivially, $C_{t}\left(\theta_{j}\right)$ attains an optimal value for $\theta_{j}=0$ or $\theta_{j}=1$. Therefore, $\hat{\theta}_{j} \in\{0,1\}$ and our proof is completed by contradiction.

Theorem 3 implies that each user $k, k=1, . ., n$ is either assigned to the WiMAX cell or to its WiFi hotspot. Thus, no load sharing between RATs is required which avoids expensive repetitive transfers between RATs.

\subsection{Algorithm for the Global Approach}

The global optimal solution for Problem $(\mathcal{P})$ can be directly computed by using an exhaustive search algorithm. Such an algorithm computes the minimum of the objective function over the set of vertices of the polytope. However, the time complexity of an exhaustive search algorithm is exponential in the number of hybrid users $n$, as given by proposition 4 .

Proposition 4. An exhaustive search algorithm that computes a global optimal solution to Problem $(\mathcal{P})$ is in $\mathcal{O}\left(2^{n}\right)$.

Proof. Theorem 3 implies that the optimal solution to Problem $(\mathcal{P})$ is obtained on the vertices of the polytope. Therefore, the objective function should be evaluated on each of the vertices, corresponding to the points where $\theta_{k}=0$ or $\theta_{k}=1$ for each $i=1, . ., n$. The cardinality of the set of vertices is given by $2^{n}$, hence the time complexity for computing the minimum value of the function over this set is in $\mathcal{O}\left(2^{n}\right)$.

\section{Numerical Results}

We consider a WiMAX cell containing three WiFi hotspots. According to the values in [1] and [2], the sets of available peak rates are given by $\chi_{k, 0}=\{7.9,16.3,24.4,32.5,48.8,65.1,73.2\}$ Mbits/s and $\chi_{k, h(k)}=\{1,2,5,11,18,36,54\}$ Mbits/s for WiMAX and WiFi respectively. Three mobile users are already present in the cell with exclusively WiMAX coverage and random achievable rates. Hybrid users arrive in sequence in the system and choose uniformly one of the three WLANs and one of the achievable rates in both RATs. In [11], there are two versions of the replicator algorithm: a synchronous version where players update simultaneously their strategy; and an asynchronous version, where each player updates its strategy randomly according to some probability of its own.

We depict in Figure 2 the probabilities $\theta_{k}$ as a function of the number of iterations in both versions of the replicator dynamics for the individual approach. We can see that the synchronous version is almost twice as fast as the asynchronous version because players update more frequently their strategies. More importantly, we can see that players' strategies converge to either 0 or 1 opting for one single RAT instead of load balancing its traffic between RATs. We recorded this behavior through the extensive simulations we performed and it seems to be a general trend which will relieve the system from costly traffic splitting between the different RATs.

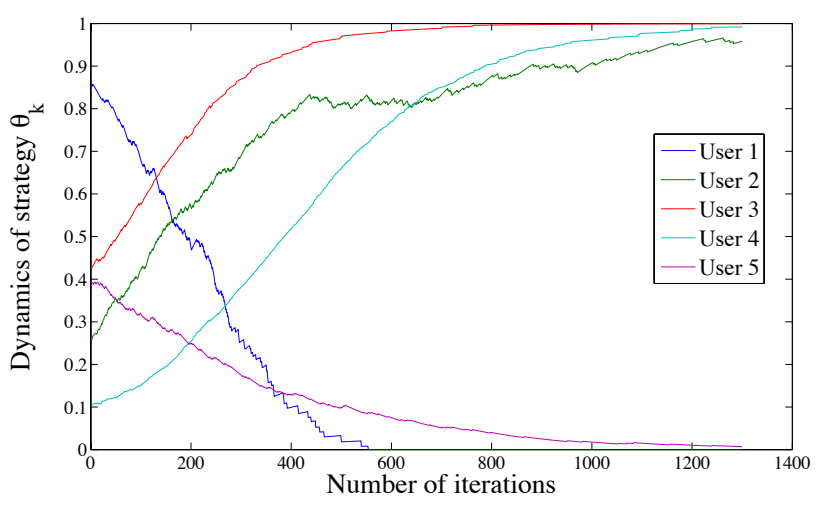

(a) Synchronous Replicator

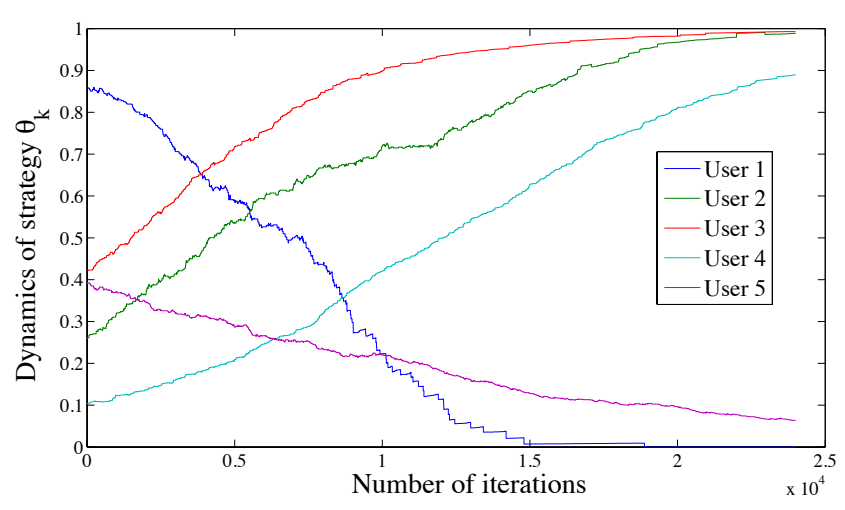

(b) Asynchronous Replicator

Figure 2: Replication Dynamics: strategy updates for 5 random users

We illustrate in Figure 3, the mean time necessary to send a data unit for all hybrid users as a function of the total number of mobile users present in the system for both the global approach in 4 and the individual approach (using our iterative algorithm in 3.1.2 and the synchronous replicator dynamics). We see that the numerical values given by the two algorithms governing the individual approach are pretty close. Furthermore, we notice an expected improvement in the global approach in comparison with the individual approach, especially for large number of hybrid users. 


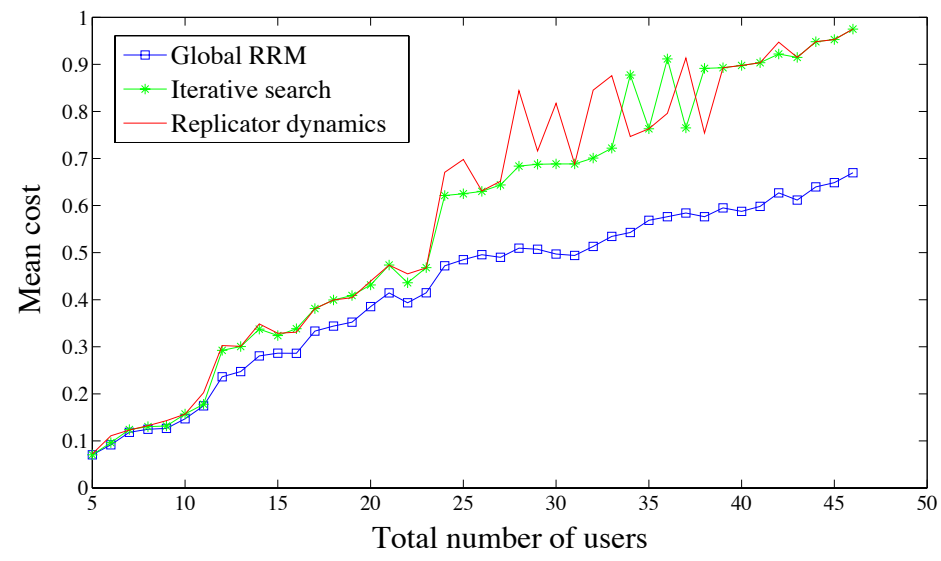

Figure 3: Comparison: Centralised and Distributed Approach

\section{Conclusion and Future work}

In this paper, radio resources are astutely allocated in a hybrid WiMAX/WiFi network by following two different approaches. In the individual approach, users selfishly aim to minimize their cost by playing a non-cooperative game. For that RRM game, an iterative algorithm is used to compute Nash equilibriums and a distributed algorithm to achieve them in a realistic fully distributed scenario. In the global approach, the system endeavors to allocate resources in a way to satisfy all users by minimizing the total network cost. Numerical comparisons between both approaches were conducted. In future work, for the individual approach, we need to examine the issue behind the non-uniqueness of NE in this setting: how to identify the most efficient equilibriums and how to implicate the system to enforce mobile users to choose such equilibriums. For the global approach, we need to examine heuristic methods.

\section{Appendix}

\subsection{Proof of convergence for dynamic replicator}

Index $x$ (resp. $\bar{x}$ ) designates a WiFi hotspot (WiMAX cell). $\theta_{k}^{x}$ resp. $\left(\theta_{k}^{\bar{x}}\right)$ is the probability of sending traffic via the hotspot $h(k)$ (resp. WiMAX cell). In [11], Bournez et al. proved that the replicator dynamics algorithm converges weakly (in the sense of weak convergence for stochastic processes) towards solutions of particular Ordinary Differential Equations (ODE), corresponding to their mean-field approximations. In fact, our stochastic algorithm behaves like ordinary differential equation as follows:

$$
\frac{d \theta_{i}^{x}}{d t}=-\theta_{i}^{y}\left(C_{i}\left(e_{y}, \theta_{-i}\right)-C_{i}\left(\theta_{i}, \theta_{-i}\right)\right)
$$

where $y \in\{x, \bar{x}\}$ and $e_{y}$ means that $\theta_{i}^{y}=1$.

This equation, called the (multi-population) replicator dynamics, is well-known to have its limit points related to Nash equilibriums (through the so-called Folk's theorem of evolutionary game theory [13]). More precisely, we have the following theorem:

Theorem 5. The following are true for the solutions of Equation (12): (i) All Nash equilibriums are stationary points. (ii) All strict Nash equilibriums are asymptotically stable. (iii) All stable stationary points are Nash equilibriums.

From [11], the limit for $b \rightarrow 0$ of the dynamics of stochastic algorithms is some ODE whose stable limit points, when $t \rightarrow \infty$ (if they exist), can only be Nash equilibriums. Hence, if there is convergence for the ordinary differential equation, then one expects the replicator dynamic algorithm to reach an equilibrium. Moreover, in [6], Coucheney et al. proved that such Nash equilibriums are pure.

Let $\alpha=\prod_{i \in N} \chi_{i, \bar{x}}$. We consider the following variation:

$$
F(\theta)=\sum_{i} \frac{\theta_{i}^{\bar{x}}}{\alpha \chi_{i, x}}\left(1+n_{x}+\chi_{i, \bar{x}} T_{i, \bar{x}}\right)+\frac{\chi_{i, \bar{x}}}{\alpha} \theta_{i}^{x}\left(\frac{1}{\chi_{i, x}^{2}}+\frac{T_{i, x}}{\chi_{i, \bar{x}}}\right)
$$

along the trajectories of this ODE (12). We compute the following partial derivatives:

$$
\frac{\partial F}{\partial \theta_{i}^{\bar{x}}}(\theta)=\frac{2 \chi_{i, \bar{x}}}{\alpha \chi_{i, x}} T_{i, \bar{x}} \text { and } \frac{\partial F}{\partial \theta_{i}^{x}}(\theta)=\frac{2 \chi_{i, \bar{x}}}{\alpha \chi_{i, x}} T_{i, x}
$$


We use (13) to determine the sign of $\frac{d F(\theta(t))}{d t}$ :

$$
\begin{aligned}
& \frac{d F(\theta(t))}{d t}=\sum_{i \in N, s \in\{x, \bar{x}\}} \frac{\partial F}{\partial \theta_{i}^{s}} \frac{d \theta_{i}^{s}}{d t} \\
= & \left.-\sum_{i \in N, s \in\{x, \bar{x}\}} \frac{\partial F}{\partial \theta_{i}^{s}}(\theta) \theta_{i}^{s}\left(C_{i}\left(e_{s}, \theta_{-i}\right)-C_{i}(\theta)\right]\right) \\
= & -\sum_{i \in N} \theta_{i}^{x}\left(1-\theta_{i}^{x}\right)\left(C_{i}\left(e_{x}, \theta_{-i}\right)-C_{i}\left(e_{\bar{x}}, \theta_{-i}\right)\right)\left(\frac{\partial F}{\partial \theta_{i}^{x}}-\frac{\partial F}{\partial \theta_{i}^{\bar{x}}}\right) \\
= & -\sum_{i \in N} \frac{2 \chi_{i, \bar{x}}}{\alpha \chi_{i, x}} \theta_{i}^{x}\left(1-\theta_{i}^{x}\right)\left(C_{i}\left(e_{x}, \theta_{-i}\right)-C_{i}\left(e_{\bar{x}}, \theta_{-i}\right)\right)^{2} \\
\leq & 0
\end{aligned}
$$

Thus $F$ is decreasing along the trajectories of the ODE and, due to the nature of the learning algorithm, all solutions of the ODE (12) remain in the strategy space if initial conditions $\in[0,1]$. From (14), we know that $\frac{d F\left(\theta^{*}\right)}{d t}=0$ implies:

$$
\theta_{i}^{* x}\left(1-\theta_{i}^{* x}\right)\left(C_{i}\left(e_{x}, \theta_{-i}^{*}\right)-C_{i}\left(e_{\bar{x}}, \theta_{-i}^{*}\right)\right)^{2}=0, \forall i
$$

Such a $\theta^{*}$ is consequently a stationary point of the dynamics. Since from theorem 5 , all stationary points that are not Nash equilibriums are unstable, proposition 2 holds.

\section{References}

[1] IEEE Standard for Local and metropolitan area networks Part 16: Air Interface for Fixed Broadband Wireless Access Systems, IEEE Std., 802.16-2004 standard.

[2] Wireless LAN Medium Access Control (MAC) and Physical Layer (PHY) specifications, ANSI/IEEE Std 802.11, 1999 Edition.

[3] Niyato D., Hossain E., A Non-cooperative Game-Theoretic Framework for Radio Resource Management in 4G Heterogeneous Wireless Access Networks, IEEE Transactions on Mobile Computing, 2008.

[4] Horrich S., Elayoubi S., Ben Jemaa S., A game-theoretic model for radio resource management in a cooperative WIMAX/ HSDPA network, IEEE ICC, 2008.

[5] Kumar D., Altman E.,User-Network Association in an 802.11 WLAN and 3G UMTS Hybrid Cell: Individual Optimality, Proceedings of IEEE Sarnoff Symposium, Princeton, USA, 2007.

[6] Coucheney P. , Touati,C., and Gaujal B. Fair and efficient user-network association algorithm for multitechnology wireless networks. INFOCOM, 2009.

[7] Ibrahim M., Khawam K., Tohme S., Network-centric joint radio resource policy in heterogeneous WiMAX-UMTS networks for streaming and elastic traffic, IEEE WCNC, 2009.

[8] J. B. Rosen, Existence and uniqueness of equilibrium points for concave n-person games, Econometrica, vol. 33, 1965

[9] Martin J. Osborne and Ariel Rubinstein, A course in game theory, MIT Press, 1994; Chinese translation published by China Social Sciences Publishing House, 2000.

[10] Topkis, Equilibrium Points in Non-Zero Sum n-Person Sub-modular Games, SIAM J. Control Optim. Volume 17, Issue 6, pp. 773-787, 1979

[11] Bournez O, Cohen J., Stochastic Learning of Equilibria in Games: The Ordinary Differential Equation Method, submitted. see paper in http://webloria.loria.fr/ jcohen/i.php/Main/HDR

[12] Weibull, J.W., 1995. Evolutionary Game Theory. MIT Press, Cambridge, MA.

[13] Hofbauer, J. and Sigmund, K. (2003). Evolutionary game dynamics. Bulletin of the American Mathematical Society, 4:479-519. 